\title{
The use of facemasks to prevent respiratory infection: a literature review in the context of the Health Belief Model
}

Shin Wei $\underline{\text { Sim}}^{1}$, Kirm Seng Peter Moey ${ }^{2}$, MMed, MCFP, Ngiap Chuan $\underline{T a n}^{2,3}$, MMed, FCFPS

INTRODUCTION Acute respiratory infections are prevalent and pose a constant threat to society. While the use of facemasks has proven to be an effective barrier to curb the aerosol spread of such diseases, its use in the local community is uncommon, resulting in doubts being cast on its effectiveness in preventing airborne infections during epidemics. We thus aimed to conduct a literature review to determine the factors that influence the use of facemasks as a primary preventive health measure in the community.

METHODS A search for publications relating to facemask usage was performed on Medline, PubMed, Google, World Health Organization and Singapore government agencies' websites, using search terms such as 'facemask', 'mask', 'influenza', 'respiratory infection', 'personal protective equipment', 'disease prevention', 'compliance' and 'adherence'. Findings were framed under five components of the Health Belief Model: perceived susceptibility, perceived benefits, perceived severity, perceived barriers and cues to action.

RESULTS We found that individuals are more likely to wear facemasks due to the perceived susceptibility and perceived severity of being afflicted with life-threatening diseases. Although perceived susceptibility appeared to be the most significant factor determining compliance, perceived benefits of mask-wearing was found to have significant effects on mask-wearing compliance as well. Perceived barriers include experience or perception of personal discomfort and sense of embarrassment. Media blitz and public health promotion activities supported by government agencies provide cues to increase the public's usage of facemasks.

CONCLUSION Complex interventions that use multipronged approaches targeting the five components of the Health Belief Model, especially perceived susceptibility, are needed to increase the use of facemasks in the community. Further studies are required to evaluate the effectiveness of implemented interventions.

Keywords: facemask, Health Belief Model, prevention, respiratory infection

\section{INTRODUCTION}

While respiratory infections are not new, they are far from being a worry of the past. From seasonal flu to catastrophic outbreaks, respiratory pathogens continue to plague us to this day. People in several countries died after being infected by the severe acute respiratory syndrome (SARS) coronavirus in 2003, and more suffered from the subsequent H5N1 and H1N1 outbreaks. ${ }^{(1,2)}$ In June 2012, a novel coronavirus causing SARS-like disease - human betacoronavirus 2c EMC/2012 (HCoV-EMC) - was discovered in the Middle East. ${ }^{(3)}$ Although HCoV-EMC does not appear to be highly contagious at the moment, ${ }^{(4)}$ it is paramount for Singapore to be prepared for an outbreak.

Globally, lower respiratory tract infections are the third most common cause of death and the most common infectious cause of death. ${ }^{(5)}$ In Singapore, acute upper respiratory tract infection, which includes influenza, is the third most common reason for attendances at public primary care clinics; it accounted for $11.1 \%$ of the 4.3 million attendances in $2010 .{ }^{(6)}$ In other words, even if respiratory infections are non-epidemic, they strain healthcare systems substantially. ${ }^{(7)}$

Pharmaceutical and nonpharmaceutical measures against respiratory infections are available. Pharmaceuticals such as vaccines and antiviral medications are highly effective in eradicating respiratory infections, as evidenced in the case of smallpox. However, as vaccines and antiviral medications take time to develop and are limited in supply, they are unable to sufficiently contain an outbreak caused by new pathogens, especially in the early stages of the outbreak. ${ }^{(8-10)}$ Nonpharmaceutical interventions, on the other hand, are not only able to aid in the control of the early stages of a new outbreak, but are also useful in everyday disease prevention in the general population. ${ }^{(8,10)}$ Measures such as frequent handwashing have been proven to be effective in preventing the transmission of viral infections (odds ratio [OR] 0.45). ${ }^{(11-13)}$ Nonpharmaceutical interventions are a cheap and noninvasive method to reduce mortality and morbidity from respiratory infections.

Aside from handwashing, the use of facemasks is also valuable in infectious disease control, especially in circumventing droplet transmission. ${ }^{(14,15)}$ For example, the effectiveness of surgical masks and N95 masks in blocking the transmission of SARS are $68 \%$ and $91 \%$, respectively. ${ }^{(7)}$ Facemasks, when fitted properly, effectively disrupt the forward momentum of particles expelled from a cough or sneeze, preventing disease transmission.

${ }^{1}$ Yong Loo Lin School of Medicine, National University of Singapore, ${ }^{2}$ SingHealth Polyclinics, ${ }^{3}$ Duke-NUS Graduate Medical School, Singapore

Correspondence: MS Shin Wei Sim, Medical student, Yong Loo Lin School of Medicine, National University of Singapore, NUHS Tower Block, Level 11, 1E Kent Ridge Road, Singapore 119228. simshinwei@gmail.com 
Even if the facemasks are ill-fitting, they are still able to interrupt the particles and airborne viruses sufficiently, such that these pathogens do not reach the breathing zones of people nearby. ${ }^{(16,17)}$

Outside the hospital environment, the effectiveness of facemasks in containing the spread of airborne diseases in the general population has been diminished largely due to improper use and lack of user compliance. ${ }^{(7)}$ An Australian study showed that among the three methods used to handle an influenza pandemic - vaccination, isolation and mask-wearing - willingness to comply with mask-wearing was the lowest. ${ }^{(18)}$ Another Australian study found that while adherence to mask-wearing significantly reduced the risk for influenza-like infections, less than $50 \%$ of the participants in their study wore facemasks regularly. ${ }^{(19)}$ In a study conducted in Singapore during the SARS outbreak, only $4 \%$ of the respondents had worn a facemask in the preceding three days. ${ }^{(20)}$ This highlights a need to uncover the determinants of mask-wearing, in order to identify the issues and overcome the barriers associated with mask-wearing compliance. The present literature review aims to evaluate these determinants and provide a framework for future interventions directed at increasing facemask usage as an effective public health measure to curb airborne infectious disease outbreaks.

\section{METHODS}

The search for relevant studies and reviews were performed using electronic journal databases, such as PubMed, Medline, Google and Google Scholar. The following keywords were used in different combinations: 'facemask', 'mask', 'surgical mask', 'PPE' or 'personal protective equipment', 'disease prevention', 'respiratory infection', 'infection', 'influenza', 'compliance', and 'adherence'. A search was also carried out on the World Health Organization website, and Singapore's government agencies (e.g. the Ministry of Health of Singapore and Singapore Health Promotion Board) websites. Studies cited by selected articles were also reviewed.

We retrieved 138 articles written in the English language, published between November 2003 and December 2012, including systematic reviews, qualitative studies, cross-sectional studies and prospective, randomised trials. The retrieved articles were then assessed for relevance. Published studies and reviews that explored reasons for compliance and/or noncompliance with mask-wearing, and literature covering factors affecting facemask use in the community and hospital settings were included, with priority given to those conducted in the community setting. Using the aforementioned criteria, a total of 51 articles were eventually selected for the present literature review.

The Health Belief Model (HBM) is a health behaviour change and psychological model developed by Rosenstock in 1966 to predict behavioural response to treatment received by acutely or chronically ill patients. ${ }^{(21)}$ It consists of four components - perceived susceptibility, perceived severity, perceived barriers and perceived benefits. With the evolving evidence generated within the health community about the role knowledge and perception play in personal responsibility, the use of $\mathrm{HBM}$ to predict more general health behaviours (e.g. the behaviour of the public in their use of facemasks in the community) has been more extensive in recent years. A modified version of the HBM that has an additional component (i.e. cues to action) was proposed by Tang et al. ${ }^{(22)}$ This fivecomponent HBM was used to present the results of the present review. HBM allows the organised classification of the determinants of mask-wearing behaviour. At the same time, it is also able to show the interdependent relationship shared among its components. HBM provides a clear frame-work for planning interventions and has been widely used to explain other forms of preventive behaviour and plan prevention programmes.

\section{RESULTS}

From our literature review, we found that the use of facemasks was associated with the following demographics:

1. Age: In Australia, a telephone survey by Taylor et al found that younger people (aged 16-34 years) were less willing to wear facemasks. ${ }^{(18)}$ In that study, willingness to comply with mask-wearing was measured by asking respondents how willing they would be to wear a facemask. Responses were coded on a five-point Likert scale, with options ranging from 'not at all' to 'extremely willing'. Tang et al evaluated factors influencing the use of facemasks to prevent the transmission of SARS in Hong Kong via telephone surveys, and found that participants aged 50-59 years were most likely to wear facemasks $(68 \%)$, while those aged 19-29 years were least likely to wear facemasks $(52.8 \%){ }^{(22)}$ In that study, ${ }^{(22)}$ compliance with mask-wearing was measured by asking participants to specify how frequently (in the past week) they wore facemasks to prevent SARS transmission. These two studies suggest that younger people were less likely to comply with mask-wearing.

2. Marital status: In the same study by Tang et al, a higher level of compliance with mask-wearing was also found among people who were married. ${ }^{(22)}$ This finding is supported by the study by Taylor et al, which found that individuals who were never married had lower compliance levels. ${ }^{(18)}$ However, the authors of that study explained that marital status was potentially a confounder as the likelihood of a person getting married increases with age. As such, it may not be marital status that predicted mask-wearing adherence, but age itself.

3. Educational level: Our literature review revealed that higher education was positively associated with mask-wearing behaviour. In a survey conducted among traditional market workers and shoppers in Taiwan, participants with a senior high school education or higher were found to be more likely to wear facemasks (adjusted OR 6.86). ${ }^{(23)}$ This trend was echoed 
by the results of telephone surveys conducted in Hong Kong by Lau et al,(24) and in Australia by Taylor et al. ${ }^{(18)}$

4. Gender: In several studies, it was observed that women were more likely to don facemasks than men. During the SARS outbreak in Hong Kong, women were reported to be more likely to wear facemasks to prevent SARS (OR 1.810, 95\% Cl 1.445-2.268). ${ }^{(22)}$ This was similarly observed during the $\mathrm{H} 1 \mathrm{~N} 1$ outbreak, with women found to be more likely to wear facemasks regularly in public areas (OR 1.94, p < 0.001) and when going out while experiencing influenza-like symptoms (OR 2.44, $\mathrm{p}<0.001) .{ }^{(25)}$ In a study of two metro stations in Mexico City, it was observed that more females wore facemasks during the H1N1 outbreak. ${ }^{(26)}$ In that study, the authors stated that this finding is consistent with the higher rate of risk-taking behaviour among men when compared to women in many situations.

Conversely, the survey conducted among traditional Taiwanese market workers and shoppers found that the adherence of workers and shoppers to avian influenza (Al) preventive behaviour, such as wearing facemasks and washing hands after contact with poultry, was not significantly affected by gender. ${ }^{(23)}$ Taylor et al also found that gender had no statistically significant effect on mask-wearing compliance. ${ }^{(18)}$ In the latter study, however, the demographics of the study's sample population were not available for further comment.

5. Ethnicity: Ethnicity may have a role in mask-wearing compliance. An Australian study by Maclntyre et al found that Caucasians appeared to be more likely to wear facemasks, ${ }^{(19)}$ and a cross-sectional survey conducted in the Singapore military by Yap et al found that Malays were more likely to practise preventive behaviour (e.g. mask-wearing and hand-washing) than other ethnic groups. ${ }^{(27)}$

6. Location of residence: The articles reviewed also suggested a possible association between the location of residence and mask-wearing compliance. Taylor et al found that people living in rural areas were more likely to wear facemasks. ${ }^{(18)}$ The study conducted in Taiwan found that people living in northeastern Taiwan were more likely to practise $\mathrm{Al}$ preventive measures compared to those living in central Taiwan (adjusted OR 6.01). ${ }^{(23)}$

In the points below, we present the findings of our literature review according to the aforementioned five components of HBM:

1. Perceived susceptibility: Perceived susceptibility refers to the extent to which one believes he or she is at risk of contracting an infectious disease. It has been well established as a major force driving adherence to mask-wearing, with a higher perception of susceptibility being linked to higher compliance with maskwearing. ${ }^{(2,22,24-26,28-30)}$ Tang et al found that individuals who personally felt very vulnerable to contracting SARS were 2.5 times more likely to wear facemasks, ${ }^{(22)}$ and Lau et al found that shortly after an imported case of H1N1 to Hong Kong, increasing numbers of confirmed H1N1 cases was one of the two most commonly mentioned factors that affected a respondent's decision to use facemasks in public places (49.3\%). . $^{(31)}$ Lau et al found that a few months after the start of the outbreak, respondents with symptoms of influenza-like illness (ILI) were more likely to wear facemasks when going out if they had been exposed to live birds or poultry within the last three months (OR 4.84, 95\% $\mathrm{Cl} 1.16-20.19, \mathrm{p}<0.05)$ or if they perceived $\mathrm{H} 5 \mathrm{~N} 1$ to have symptoms similar to those of influenza (OR 4.79, 95\% Cl 1.4915.39, $\mathrm{p}<0.01) .{ }^{(31)}$ In the Taiwan traditional market survey, Kuo et al found that Al preventive behaviour was more likely to be performed by participants who had been informed of local Al outbreaks (adjusted OR 2.24). ${ }^{(23)}$ Higher risk perception scores concerning influenza were also associated with maskwearing behaviour in a study of a Hispanic population in Northern Manhattan, which had predominantly female participants. ${ }^{(29)}$

2. Perceived benefits: In the present context, perceived benefits refer to how effective facemasks are believed to be in preventing the spread of diseases by the community and/or individual. Positive correlations have been found to exist between perceived benefits and the likelihood that an individual would be willing to wear a facemask. . $22,24,25,32)$ In Hong Kong, Tang et al found that individuals who had strong beliefs in the effectiveness of wearing facemasks were 1.4 times more likely to wear facemasks during SARS. ${ }^{(22)}$ Lau et al found that during the H1N1 outbreak, those who perceived wearing facemasks in public areas to be a very effective self-protection measure were more likely to wear facemasks (OR 1.90, p < 0.001). ${ }^{(25)}$ In the study of the Hispanic group with predominantly female participants, increased perception of the effectiveness of mask-wearing was also associated with mask-wearing behaviour. ${ }^{(29)}$

3. Perceived severity: Perceived severity refers to how much an individual fears a disease or an outbreak, and how worried he/she is that his/her place of residence would become a quarantine city. ${ }^{(22)}$ During the early SARS outbreak in Hong Kong, the results of a telephone survey showed that perceived susceptibility, perceived benefits and cues to action were significant predictors of facemask use. Perceived severity was not found to be a significant predictor of the use of facemasks. ${ }^{(22)}$ The authors, Tang et al, attributed this finding to the individuals' underestimation of the disease's potential to become a global epidemic. During the early stage of the local outbreak, the disease mainly affected healthcare workers, a few index patients, and the close contacts of the two mentioned groups. The dire outcome of the disease with increasing prevalence and fatalities could have been overlooked.(22)

In contrast, shortly after an imported case of H1N1 to Hong Kong, Lau et al found that reports of deaths of local H1N1 patients affected the decision to use facemasks in public areas 
$(13.1 \%){ }^{(25)}$ The authors found that over the duration of the H1N1 outbreak, those who perceived that H1N1 infection had a very high fatality rate were more likely to wear facemasks regularly in public areas (OR 1.64, p < 0.01). ${ }^{(25)}$ However, perceived severity did not affect the wearing of facemasks when going out in cases of ILI. Although $88.7 \%$ of the respondents had initially stated they would wear facemasks when having symptoms of ILI, only $36.6 \%$ of respondents self-reported that they had often or always worn facemasks in public areas when having symptoms of ILI, a few months after the outbreak. ${ }^{(33)}$ Among those with symptoms of ILI, respondents were found to be more likely to wear facemasks when going out if they perceived the impact of bird-to-human $\mathrm{H} 5 \mathrm{~N} 1$ to be worse than that SARS (OR 3.21, 95\% Cl 1.21-7.91, $\mathrm{p}<0.05)$.

In a study by Syed et al, the authors described their journey from the United Kingdom to Thailand during the 2003 SARS scare. ${ }^{(34)}$ They observed that the prevalence of mask-wearers in Thailand rose during the outbreak and hypothesised that the perceived severity of SARS as a threat to the economy and public health was associated with the increased social acceptance of facemasks. ${ }^{(34,35)}$ In Australia, Taylor et al found that individuals who reported to be extremely or very concerned for self and/or family in the event of a pandemic influenza were more willing to wear facemasks (OR 1.94, p < 0.001). ${ }^{(18)}$ A 2009 study by Eastwood et al, in which interviews were conducted with adult Australians, found that an increase in anxiety was associated with an increase in the willingness to wear facemasks. ${ }^{(15)}$

A lack of adequate knowledge about a disease may also hinder mask-wearing compliance. The street survey in Taiwan traditional markets by Kuo et al found that individuals who lacked correct knowledge about the fatality rate of avian influenza were four times less likely to practise appropriate Al preventive behaviour, including mask-wearing. ${ }^{(23)}$ Factors associated with correct Al preventive behaviour included having correct knowledge about the fatality rate of $\mathrm{Al}$ (adjusted OR 4.18) and having knowledge of severe cases of Al (adjusted OR 2.13).

In a cross-sectional survey conducted in Singapore in 2009, influenza patients were shown to be more compliant with mask-wearing than the general population, contacts of influenza patients and healthcare workers. ${ }^{(27)}$ Healthcare workers and contacts of influenza cases were, however, more compliant with preventive measures (e.g. mask-wearing and vaccination) than the general population. This was likely because these individuals, unlike the general population, had more first-hand experience with influenza. ${ }^{(27)}$ The survey also found that there was a strong correlation between the level of knowledge about the disease and likelihood of practising preventive behaviour. ${ }^{(27)}$

4. Perceived barriers: In the present context, perceived barriers refer to factors that potentially prevent or discourage individuals from using facemasks. In a cluster-randomised trial by Maclntyre et al, households in Australia were given either (a) P2 masks, which are the equivalent of N95 masks; (b) surgical masks; or (c) no facemasks. ${ }^{(19)}$ Households given facemasks (either P2 masks or surgical masks) were instructed that the facemasks were to be worn at all times in the presence of a child infected with respiratory infection. In that study, more than half of the mask-wearers voiced concerns about the discomfort associated with mask-wearing. Other barriers to compliance were that the children of mask-wearers disliked their parents wearing facemasks, forgetfulness, ill fit of the facemasks provided, and the impracticality of wearing facemasks in certain situations, such as during meal times and while sleeping. Other than that, the study found that participants with three or more adults in the house were less likely to wear facemasks. ${ }^{(19)}$

Ferng et al's study, which aimed to evaluate the barriers to mask-wearing among Hispanic households of Northern Manhattan, involved 'think-aloud' activities and focus group discussions. ${ }^{(29)}$ The study found that discomfort resulted from poor fit of the facemasks, which was due to certain facial structures of the wearer. For instance, facemasks tend to slide down the faces of wearers with low cheekbones and small nose bridges. In addition, it was reported that ill individuals might find it unpleasant to wear facemasks, especially if the user suffers from nasal congestion or rhinorrhoea. Facemasks also tend to become damp after a period of time in warm environments, further contributing to the discomfort of mask-wearing. ${ }^{(29)}$ In a study by Weiss et al, the authors identified four barriers that may discourage individuals from wearing facemasks: (a) discomfort, especially in hot weather; (b) the presence of chronic lung disease; (c) inconvenience, due to the need to remove facemasks when eating or drinking; (d) young age, as facemasks are generally not designed for children, and even if they were, children were found to be unlikely to wear them for long periods of time. ${ }^{(36)}$

In Hong Kong, Tang et al found that perceived barriers was not a significant factor affecting mask-wearing compliance during SARS. ${ }^{(22)}$ During epidemics, perceived susceptibility and perceived severity outweighed the effect perceived barriers had with regard to facemask use. The authors postulated that since facemasks are relatively easy to put on and remove, as well as cheap and accessible (except during the early stages of the SARS outbreak), they present as less of a barrier, despite the discomfort and inconvenience associated with their use.

The cost of facemasks was a factor that was not thoroughly covered in any of the studies that investigated the determinants of mask-wearing compliance. Nevertheless, monetary cost to the individual has been found to affect compliance with other health measures. In a study by Campbell et al, which studied the effect of asthma medication price changes on asthma medication use, the authors found that even a slight increase in medication costs led to a severe decline in medication use. ${ }^{(37)}$ In Taiwan, it was reported that N95 masks that originally 


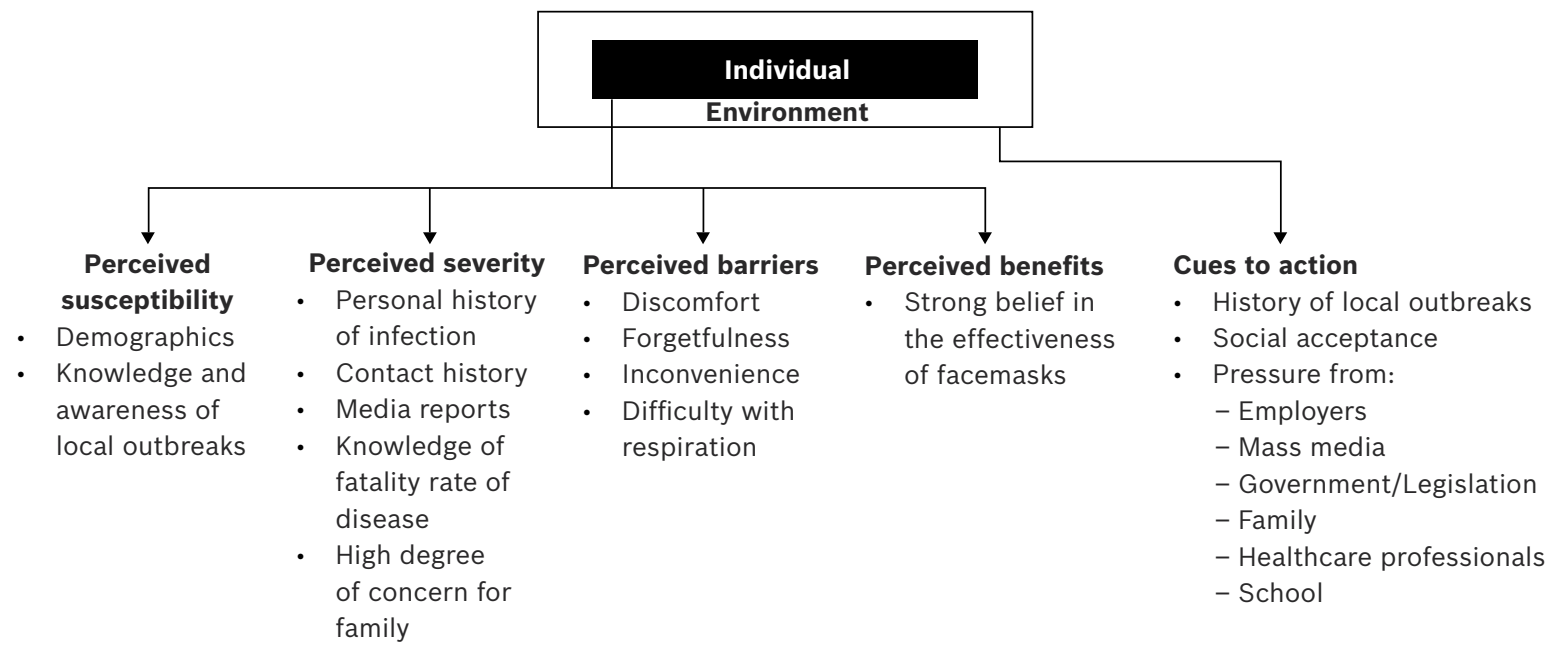

Fig. 1 Flow chart shows the factors that influence compliance with mask-wearing, in the context of the Health Belief Model.

cost 85 cents each were sold for USD 20 each during the SARS outbreak in 2003. ${ }^{(38)}$ However, the report did not elaborate on the impact of cost on an individual's decision to don masks, which may be significant, particularly during outbreaks when the demand for masks may outstrip the supply.

5. Cues to action: Other than individual beliefs, one's choice of whether or not to comply with mask-wearing is also impacted by environmental factors. The family, society, media and government play important roles in providing cues to encourage people to take preventive measures (e.g. the use of facemasks), particularly during a disease outbreak in the community. In the Hong Kong survey conducted by Tang et al during the SARS outbreak, participants were asked to rate the degree to which the local government and their family members encourage them to don facemasks. ${ }^{(22)}$ Participants who were more aware of environmental cues were found to be 2.4 times more likely to wear facemasks.

Japan is a country well-known for its prevalent use of facemasks in the community, even during periods when outbreaks are not prevalent. Burgess et al theorised that the spate of epidemics in Japan (e.g. the avian flu in 2004 and the swine flu in 2009) played key roles in heightening residents' perceived susceptibility, triggering a surge in the use of facemasks and perpetuating the use of facemasks in the local community. ${ }^{(2)}$ Burgess et al also attributed part of Japan's mask-wearing culture to the fact that some employees in Japan experience pressure from their bosses to don facemasks. ${ }^{(2)}$ In other words, in cases where the failure to don facemasks adversely affected one's income and/or employment, compliance with mask-wearing seemed to improve. This is further supported by the study conducted in Mexico City, where the authors observed that taxi drivers who wore facemasks during the H1N1 outbreak were preferred by people hailing taxis, possibly contributing to the higher rate of facemask use among taxi drivers than bus drivers. ${ }^{(26)}$
Social acceptance was another key factor in increasing or decreasing the rate of compliance. In Japan, a street survey showed that most Japanese agreed that healthy individuals are being 'respectful' towards one's health in a positive way by wearing facemasks. Hence, mask-wearing had not only become a normalised practice, but a desirable one. ${ }^{(2)}$ In a qualitative study involving students of an Australian university, Seale et al established embarrassment to be a contributing factor to noncompliance. ${ }^{(32)}$ Respondents in the study voiced concerns about mask-wearers being perceived as 'dangerous' and attracting unwanted attention. ${ }^{(32)}$ Syed et al observed how peer pressure could significantly influence the use of facemasks. ${ }^{(34)}$ Passengers who arrived in Bangkok without facemasks, bought and wore them before boarding their next flight after witnessing all staff at the Bangkok airport donning facemasks. This occurred despite the fact that there was no clear recommendation for these passengers to do so. ${ }^{(34)}$

Pressure from the media, in the form of impactful advertising campaigns (e.g. by mask manufacturers) and the appearance of news reporters donning facemasks on national television during epidemics, has been shown to contribute to the high levels of adherence to mask-wearing in Japan. (2) Such advertisements, which portray the mask-wearing individual as the ideal employee and family person, may have helped to mould the mindset of the Japanese. The increase in the use of facemasks in Thailand during the SARS outbreak was also partly attributed to media influence. ${ }^{(34)}$

Official public policies have been shown to have a significant role in promoting the use of facemasks. The frequency of facemask usage in the community has been shown to increase after governments and public health officials promoted the use of facemasks, especially during periods of disease

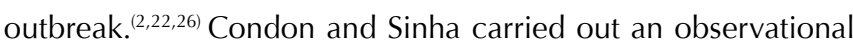
study during the severe third wave of the H1N1 epidemic in Mexico City, during which there was paucity of official publicity regarding the outbreak. ${ }^{(26)}$ The authors observed that the paucity 
of official publicity resulted in the scarce use of facemasks (quantification of the use of facemasks was based on the computation of the number of facemask users in the subway). This contrasted with the higher prevalence of facemask users during the first wave of the H1N1 outbreak, during which the local official agencies encouraged the use of facemasks, and mandated drivers in the public transport system to wear facemasks and gloves. ${ }^{(26)}$ In the hospital setting, Chor et al found that differences in the guidelines stipulated by the health authorities of different countries correlated with the differences in facemask usage rates observed in the respective hospitals of the countries. ${ }^{(39)}$

Other than the environmental factors mentioned, Burgess et al found that the reason why some Japanese donned facemasks was due to perceived pressure from one's family members, doctors and school, suggesting that these environmental factors may also aid in improving compliance with mask-wearing. ${ }^{(2)}$ A summary of the five HBM components that determine compliance with mask-wearing is presented in Fig. 1.

\section{DISCUSSION}

Based on our literature review, we observed that perceived severity was not a significant factor influencing mask-wearing compliance in the study by Tang et al. ${ }^{(22)}$ However, Tang et al reported that as the study was carried out during the early stages of the SARS outbreak in Hong Kong, there was a relative lack of understanding of the disease and its potential to become a serious threat to the community at that time. In other words, there is the possibility that perceived severity may become a significant predictor of facemask usage with increased awareness of the disease.

Studies investigating other forms of preventive behaviour and disease control measures, such as handwashing and covering one's mouth when coughing or sneezing, have findings that are similar to those of the aforementioned studies evaluating the determinants of mask-wearing compliance. These studies showed that the following were significant predictors of improved compliance with other forms of preventive behaviour: higher perceived susceptibility, higher perceived severity, higher perceived benefits, older age, female gender, higher education level, presently or previously married, higher level of awareness of a disease or health measure, and provision of information by health professionals. ${ }^{(18,24,40-42)}$

With regard to gender, it has been postulated that women are generally less willing to take risks, and are thus more compliant with preventive behaviour than their male counterparts. Such differences in risk-taking have been attributed to evolutionary differences between the two genders. ${ }^{(43)}$ In order to ensure that they are able to care for their offspring, females tend to avoid risky activities. In contrast, males need to take more risks in order to fulfil their roles - to hunt, to protect their mates and offspring, and to increase their chances of attracting a mate.
The trend that individuals with a higher level of education are more likely to be compliant with preventive behaviours may be explained by these individuals' increased awareness of diseases and disease consequences, which allow them to have a more severe perception of the risks associated with noncompliance. As these individuals might also have a better understanding of public health measures and their effectiveness, and consequently a higher perception of the benefits of these preventive behaviours, they are more likely to be compliant to these measures.

A possible reason as to why individuals who are currently or previously married have been found to be more adherent to preventive behaviour than those who have never been married is that those who are, or have been, married feel responsible, and perhaps anxious, for the health of not just themselves, but also their loved ones (e.g. their spouse and children). This might have led to increased perceived severity and susceptibility, resulting in increased compliance.

Although it is evident from the literature reviewed that cues to action played a crucial role in influencing mask-wearing compliance, it is important to note that this may only hold true when there is sufficient trust between the individual and the environmental factors. In the H1N1 study by Condon and Sinha in Mexico City, the effectiveness of mandatory and voluntary public health measures set by the government was diminished by the lack of trust in the government. ${ }^{(26)}$ Likewise, one can expect healthcare professionals to have little or no impact in influencing a person's decision to comply with public health measures if the person does not trust or value the advice given by these healthcare professionals.

With the findings mentioned, we propose that healthcare policy-makers intervene using factors derived from the multipronged HBM, which focuses on increasing the public's perceived susceptibility, perceived severity and perceived benefits; decreasing perceived barriers; and increasing the cues to appropriate actions. From our literature review, perceived susceptibility appears to be the most significant factor influencing compliance with mask-wearing. To increase the public's perceived susceptibility, there should be increased education on respiratory infections (e.g. mode of transmission, serious implications of infection and preventive measures). ${ }^{(15)}$ There should also be timely updates about outbreaks. By making information about new outbreaks more easily available to the public, and at an earlier time, awareness and understanding of the emergent epidemic would improve. This would, in turn, increase the public's perceived susceptibility and severity, hence potentially improving compliance. In addition, promoting the use of facemasks and educating the public about mask-wearing as an effective tool in disease prevention may also help to increase the public's perceived benefits, which may then also contribute to increasing mask-wearing compliance.

Healthcare professionals also play an important role in reiterating the preventive measures provided by official 
healthcare agencies and in motivating their patients toward appropriate behaviour change. Good doctor-patient relationships based on trust and mutual respect is a key element to effecting change. In a telephone survey conducted in 2007, it was found that Australians were more likely to seek information about the outbreak from general practitioners and other healthcare professionals than the public health department. ${ }^{(44)}$ The participants in that study were reviewed two years later, and the results obtained were consistent with that of the 2007 telephone survey. ${ }^{(15)}$ Thus, it is not only important to keep healthcare providers updated on outbreaks and methods of disease prevention, it is also crucial to encourage healthcare providers to play an active role in educating the public on such topics. Aside from sharing facts about disease transmission and prevention, Yap et al proposed the use of narratives and case illustrations by healthcare workers, as this may aid in improving compliance with preventive measures like mask-wearing since it would increase the public's perceived susceptibility, perceived severity and perceived benefits. ${ }^{(27)}$

Schools have a role to play too. ${ }^{(15)}$ They can help inculcate mask-wearing habits in the young, just as how proper handwashing habits are taught to children in Singapore in kindergartens. By instilling these habits from a young age, we may be able to cultivate a generation of individuals who grow up perceiving mask-wearing as a normalised, socially acceptable practice, rather than an odd or embarrassing one.

As mentioned earlier, the effect that the cost of facemasks has on mask-wearing compliance has not been explored. Therefore, further studies should be conducted to investigate whether any significant relationship exists between the cost of facemasks and an individual's willingness to wear facemasks. If such studies show that cost has a strong correlation with mask-wearing compliance, it may be valuable to consider various ways in which the cost of facemasks can be lowered for the individual, such as developing reusable facemasks. ${ }^{(36)}$

The discomfort experienced with the use of facemasks is an issue that needs to be further investigated. Although discomfort was raised as a concern by mask-wearers in the study by Macintyre et al, details of what contributes to discomfort were not elicited. ${ }^{(19)}$ On the other hand, in a study by Ferng et al, the significance of the specific factors contributing to facemask discomfort was not established.(29) Thus, more research is required to establish the significance discomfort has on mask-wearing compliance, to uncover the specific factors contributing to discomfort, and to determine the impact that these specific factors have on discomfort. To improve user compliance, Jefferson et al suggested designing new facemasks that increase comfort. ${ }^{(7)}$ Aside from comfort, respondents from the study by Burgess et al also expressed that they wished to see trendier and more user-friendly facemasks. ${ }^{(2)}$ Thus, facemasks that are easy to wear, comfortable and attractive in appearance may encourage more individuals to don them.
Table I. Summary of the literature review on the use of facemasks to prevent respiratory infection.

- Facemasks play an important role in the prevention and control of infectious respiratory disease transmission. However, compliance issues reduce the effectiveness of mask-wearing as a public health measure.

- Factors that influence mask-wearing compliance can be categorised according to the five components of the Health Belief Model - perceived susceptibility, perceived benefits, perceived severity, perceived barriers and cues to action.

- Perceived susceptibility appears to be the most significant factor determining compliance. Low compliance rates are often observed when risk perception is low (e.g. no outbreaks imminent).

- A concerted effort by healthcare policy makers and workers, educational institutions and the mass media is paramount in improving mask-wearing compliance. Efforts should be made to decrease perceived barriers, and to increase perceived benefits, perceived severity, cues to action, and in particular, perceived susceptibility.

Although the HBM was chosen for our literature review due to its strengths, it is not without limitations. The definitions of each of the five components are rather broad, and thus some factors affecting mask-wearing compliance may overlap and fall into more than one HBM component. This may cause difficulty in the identification of the most significant component influencing mask-wearing compliance when comparisons are made among the five components.

Facemasks play a pivotal role in the prevention and control of infectious respiratory disease transmission. However, compliance with its usage is influenced by multiple factors, which with the use of the five components of HBM, can be framed for easy referencing. To enhance the widespread use of facemasks, which is required in the advent of an epidemic, further studies are needed to evaluate the effectiveness of interventions targeted at increasing mask-wearing. Multipronged approaches should be used to address the factors that influence compliance with mask-wearing. The main points of this review are summarised in Table I.

\section{REFERENCES}

1. World Health Organization. Global and Alert Response (GAR), Disease outbreaks by year [online]. Available at: http://www.who.int/csr/don/ archive/year/en/index.html. Accessed April 20, 2013.

2. Burgess A, Horii M. Risk, ritual and health responsibilisation: Japan's 'safety blanket' of surgical face mask-wearing. Sociol Health Illn 2012; 34:1184-98.

3. Müller MA, Raj VS, Muth D, et al. Human corona virus EMC does not require the SARS-coronavirus receptor and maintains broad replicative capability in mammalian cell lines. MBio 2012; 3:e00515-12.

4. Chan JF, Li KS, To KK, et al. Is the discovery of the novel human betacoronavirus 2c EMC/2012 (HCoV-EMC) the beginning of another SARS-like pandemic? J Infect 2012; 65:477-89.

5. Wiemken TL, Peyrani P, Ramirez JA. Global changes in the epidemiology of community-acquired pneumonia. Semin Respir Crit Care Med 2012; 33:213-9.

6. Ministry of Health, Singapore. Top 4 Conditions of Polyclinic Attendances [online]. Available at: https://www.moh.gov.sg/content/ moh_web/home/statistics/Health_Facts_Singapore/Top_4_ Conditions_of_Polyclinic_Attendances.html. Accessed August 9, 2012. 
7. Jefferson T, Foxlee R, Del Mar C, et al. Physical interventions to interrupt or reduce the spread of respiratory viruses: systematic review. BMJ 2008; 336:77-80.

8. Aiello AE, Perez V, Coulborn RM, et al. Facemasks, Hand hygiene, and influenza among young adults: a randomized intervention trial. PLoS One 2012; 7:e29744.

9. Aledort JE, Lurie N, Wasserman J, Bozzette SA. Non-pharmaceutical public health interventions for pandemic influenza: an evaluation of the evidence base. BMC Public Health 2007; 7:208.

10. Kiliç S, Gray GC. Nonpharmaceutical Interventions for Military Populations During Pandemic Influenza. Turk Silahli Kuvvetleri Koruyucu Hekim Bul 2007; 6:285-90.

11. Luby SP, Agboatwalla M, Feikin DR, et al. Effect of handwashing on child health: a randomised controlled trial. Lancet 2005; 366:225-33.

12. White CG, Shinder FS, Shinder AL, Dyer DL. Reduction of illness absenteeism in elementary schools using an alcohol-free instant hand sanitizer. J Sch Nurs 2001; 17:258-65.

13. Jefferson T, Del Mar C, Dooley L, et al. Physical interventions to interrupt or reduce the spread of respiratory viruses. Cochrane Database Syst Rev 2010; (1):CD006207.

14. Furuya $\mathrm{H}$. Risk of transmission of airborne infection during train commute based on mathematical model. Environ Health Prev Med 2007; 12:78-83.

15. Eastwood K, Durrheim D, Francis JL, et al. Knowledge about pandemic influenza and compliance with containment measures among Australians. Bull World Health Organ 2009; 87:588-94.

16. van der Sande M, Teunis P, Sabel R. Professional and home-made face masks reduce exposure to respiratory infections among the general population. PLoS One 2008; 3:e2618.

17. Tang JW, Liebner TJ, Craven BA, Settles GS. A schlieren optical study of the human cough with and without wearing masks for aerosol infection control. J R Soc Interface 2009; 6 suppl 6:S727-36.

18. Taylor M, Raphael B, Barr M, et al. Public health measures during an anticipated influenza pandemic: Factors influencing willingness to comply. Risk Manag Healthc Policy 2009; 2:9-20.

19. Maclntyre CR, Cauchemez S, Dwyer DE, et al. Face mask use and control of respiratory virus transmission in households. Emerg Infect Dis 2009; 15:233-41.

20. Quah SR, Hin-Peng L. Crisis prevention and management during SARS outbreak, Singapore. Emerg Infect Dis 2004; 10:364-8.

21. Rosenstock IM. Why people use health services. Milbank Meml Fund Q 1966; 44:94-127.

22. Tang CS, Wong CY. Factors influencing the wearing of facemasks to prevent the severe acute respiratory syndrome among adult Chinese in Hong Kong. Prev Med 2004; 39:1187-93.

23. Kuo PC, Huang JH, Liu MD. Avian influenza risk perception and preventive behaviour among traditional market workers and shoppers in Taiwan: practical implications for prevention. PLoS One 2011; 6:e24157.

24. Lau JT, Kim JH, Tsui HY, Griffiths S. Anticipated and current preventive behaviours in response to an anticipated human-to-human $\mathrm{H} 5 \mathrm{~N} 1$ epidemic in the Hong Kong Chinese general population. BMC Infect Dis 2007; 7:18.

25. Lau JT, Griffiths S, Choi KC, Lin C. Prevalence of preventive behaviours and associated factors during early phase of the $\mathrm{H} 1 \mathrm{~N} 1$ influenza epidemic. Am J Infect Control 2010; 38:374-80.

26. Condon BJ, Sinha T. Who is that masked person: the use of face masks on Mexico City public transportation during the Influenza A (H1N1) outbreak.
Health Policy 2010; 95:50-6.

27. Yap J, Lee VJ, Yau TY, Ng TP, Tor PC. Knowledge, attitudes and practices towards pandemic influenza among cases, close contacts, and healthcare workers in tropical Singapore: a cross-sectional survey. BMC Public Health 2010; 10:442.

28. Lau JT, Griffiths S, Au DW, Choi KC. Changes in knowledge, perceptions, preventive behaviours and psychological responses in the pre-community outbreak phase of the H1N1 epidemic. Epidemiol Infect 2011; 139:80-90.

29. Ferng YH, Wong-McLoughlin J, Barrett A, Currie L, Larson E. Barriers to mask wearing for influenza-like illnesses among urban Hispanic households. Public Health Nurs 2011; 28:13-23.

30. Chia SE, Koh D, Fones C, et al. Appropriate use of personal protective equipment among healthcare workers in public sector hospitals and primary healthcare polyclinics during the SARS outbreak in Singapore. Occup Environ Med 2005; 62:473-7.

31. Lau JT, Griffiths S, Choi KC, Tsui HY. Widespread public misconception in the early phase of the H1N1 influenza epidemic. J Infect 2009; 59:122-7.

32. Seale H, Mak JP, Razee H, Macintyre CR. Examining the knowledge, attitudes and practices of domestic and international university students towards seasonal and pandemic influenza. BMC Public Health 2012; $12: 307$.

33. Lau JT, Kim JH, Tsui HY, Griffiths S. Perceptions related to bird-to-human avian influenza, influenza vaccination, and use of face mask. Infection 2008; 36:434-43.

34. Syed Q, Sopwith W, Regan M, Bellis M. Behind the mask. Journey through an epidemic: some observations of contrasting public health responses to SARS. J Epidemiol Community Health 2003; 57:855-6.

35. World Health Organization. Severe acute respiratory syndrome (SARS): status of the outbreak and lessons for the immediate future [online]. Available at: http://www.who.int/csr/media/sars_wha.pdf. Accessed April 20, 2013

36. Weiss MM, Weiss PD, Weiss DE, Weiss JB. Disrupting the transmission of influenza a: face masks and ultraviolet light as control measures. Am J Public Health 2007; 97 suppl 1:S32-7.

37. Campbell JD, Allen-Ramey F, Sajjan SG, Maiese EM, Sullivan SD. Increasing pharmaceutical copayments: impact on asthma medication utilization and outcomes. Am J Manag Care 2011; 17:703-10.

38. McNeil D. Masks become a Taiwan must-have, with bootlegging and price gouging. New York Times 2003 May 22; A8.

39. Chor JS, Pada SK, Stephenson I, et al. Differences in the compliance with hospital infection control practices during the 2009 influenza H1N1 pandemic in three countries. J Hosp Infect 2012; 81:98-103.

40. Leung GM, Lam TH, Ho LM, et al. The impact of community psychological responses on outbreak control for severe acute respiratory syndrome in Hong Kong. J Epidemiol Community Health 2003; 57:857-63.

41. Di Giuseppe G, Abbate R, Albano L, Marinelli P, Angelillo IF. A survey of knowledge, attitudes and practices towards avian influenza in an adult population of Italy. BMC Infect Dis 2008; 8:36.

42. Pawlowski B, Atwal R, Dunbar R. Sex differences in everyday risk-taking behaviour in humans. Evol Psychol 2008; 6:29-42.

43. Archer J. Sex differences in aggression in real-world settings: a metaanalytic review. Rev Gen Psychol 2004; 8:291-322.

44. Eastwood K, Durrheim DN, Butler M, Jones A. Responses to pandemic (H1N1) 2009, Australia. Emerg Infect Dis 2010; 16:1211-6. 